\title{
miRNA-34a-5p downregulation of VEGFA in endometrial stem cells contributes to the pathogenesis of endometriosis
}

\author{
YING MA, YU-XIN HUANG and YAN-YING CHEN \\ Department of Gynaecology and Obstetrics, Zhujiang Hospital of Southern Medical University, \\ Guangzhou, Guangdong 510280, P.R. China
}

Received February 19, 2017; Accepted June 15, 2017

DOI: $10.3892 / \mathrm{mmr} .2017 .7677$

\begin{abstract}
Endometrial-derived stem cells (EnSCs) serve an important role in the development of endometriosis via retrograde menstruation. Abnormal expression of miRNAs in EnSCs is involved in the etiology of endometriosis, however, the mechanisms remain unclear. The aim of the present study was to investigate the expression of miR-34a-5p and VEGFA in endometrial samples from patients with or without endometriosis, and then examine the underlying mechanism of microRNA-34a-5p regulation of VEGFA in EnSCs. Endometrial samples from patients with or without endometriosis were collected, and miR-34a-5p expression in the two group s was measured using RT-PCR. Human endometrial-derived stem cells (hEnSCs) were isolated from these endometrial samples, and hEnSCs were transfected with the miR-34a-5p mimics or control miRNAs. qPCR and western blotting were performed to assess the effects of miR-34a-5p on the expression of VEGFA in hEnSCs, and cell growth was assessed by an MTT assay. miR-34a-5p was significantly downregulated in patients with endometriosis when compared with that of those without endometriosis. VEGFA expression levels in hEnSCs with an overexpression of miR-34a-5p were significantly reduced when compared with those in the negative control $(\mathrm{P}<0.01)$. In addition, the upregulation of miR-34a-5p suppressed EnSCs proliferation by targeting the $3^{\prime}$ untranslated region of VEGFA. miR-34a-5p provides a novel avenue for the understanding of the development of endometriosis, and may facilitate the development of potential therapeutics against endometriosis.
\end{abstract}

\section{Introduction}

Endometriosis is a common, and multifactorial gynaecological disorder that affects $10-15 \%$ of reproductive-age women (1).

Correspondence to: Professor Ying Ma, Department of Gynaecology and Obstetrics, Zhujiang Hospital of Southern Medical University, 253th Industrial Avenue, Guangzhou, Guangdong 510280, P.R. China

E-mail: mayingwuzhuoyi@126.com

Key words: miR-34a-5p, endometriosis, vascular endothelial growth factor (VEGF), endometrial-derived stem cells (EnSCs)
The development of this condition is endometrial tissue grows outside of the uterine cavity, which can induce varying degrees of painful symptoms and infertility in infected individuals (2). Endometriosis also has a propensity to recur and may be associated with ovarian cancer (3). The impact of endometriosis is great, likely exceeding our expectations, as it can dramatically impair the quality of life.

The pathogenesis of endometriosis was initially defined as the presence of endometrial tissues in extra-uterine cavity, including pelvic peritoneum, bladder and ureters (4). There are several hypotheses have been proposed, including lymphatic and vascular metastasis, iatrogenic direct implantation, coelomic metaplasia, embryonic rest and mesenchymal cell induction (5). Moreover, the development of endometriosis was also involved in stem cells (6). This theory postulates that the endometrial stem cells are abnormally shed during menses, where they gain access to the peritoneal cavity by retrograde menstruation and ectopic implantation. The presence of mesenchymal stem cells (MSCs) in stromal cells of ectopic endometrial tissues was reported (7). These results indicated that ectopic endometrial stem cells (EnSCs) are regarded as responsible for the pathogenesis of endometriosis.

Endometriosis has also been defined as an angiogenic disease, since the ectopic survival of endometrium requires the formation of new blood vessels. Angiogenesis, therefore, is a critical step in developing endometriotic lesions (8). Vascular endothelial growth factor (VEGF) is a member of VEGFA family, coding by a $28 \mathrm{~kb}$-long gene which is located on chromosome $6 \mathrm{p} 21.3$. It is known as a crucial regulator of angiogenesis, endothelial cells growth and migration (9). Several studies have reported that VEGFA play an important role in the angiogenesis of endometriosis (10). miRNAs such as miR-126, Let7-f, miR-27b, miR-17-92 cluster and miR-130a were identified as proangiogenic miRNAs that regulate the translation of angiogenic factors (VEGF-A) (11). miRNAs have recently emerged as an important factor in endometriosis (12). Toloubeydokhti et al (13) assessed the expression of miR-17-5p, miR-23a, miR-23b and miR-542-3p, and found that miR-23b and miR-542-3p are downregulated, whereas $\mathrm{miR}-17-5 \mathrm{p}$ is upregulated in ectopic endometrium, affecting the stability of their target genes' expression, and playing an important role in the pathogenesis of endometriosis. Moreover, miRNAs are aberrantly expressed in endometriotic stromal cells play an important role in the pathogenesis of 
endometriosis (14). miR-34a-5p is highly expressed in multiple types of cancer, which inhibited tumor angiogenesis by blocking VEGF production by directly inhibiting endothelial cell functions (15).

In this study, the aim was to investigate the expression of miR-34a-5p and VEGFA in endometriosis tissues, and analyzed the function and mechanism of miR-34a on the endometriosis stem cells (EnSCs).

\section{Materials and methods}

Endometriosis tissues. Ten endometriosis patients were diagnosed by laparoscopic surgical examination, and undergone surgical excision of endometriosis tissues. The control tissue samples were collected from 10 premenopausal patients without endometriosis. All these patients had not received any preoperative hormonal therapy or taken any medicine for at least three months. The resected tissues were minced and part of samples was stored at $-80^{\circ} \mathrm{C}$ before the total RNA and protein extraction. Written consent was obtained from each patient before the study. This study was approved by the Research Ethics Committee of the Zhujiang Hospital of Southern Medical University (Guangzhou, China).

Endometrial stem cell isolation, culture and transfection. Human endometriosis tissue was washed in PBS (Gibco, USA), minced, and digested in collagenase ( $1 \mathrm{mg} / \mathrm{ml}$, Gibco, USA) for $30-45 \mathrm{~min}$ at $37^{\circ} \mathrm{C}$ with agitation. Then, resultant cell solutions were filtered and centrifuged, and mononuclear cells were separated by Ficoll (Sigma, St. Louis, MO, USA) and washed in PBS. The isolated cells were cultured in Dulbecco's modified Eagle's medium (DMEM)/F-12 medium containing $10 \%$ fetal bovine serum (FBS) and 1\% penicillin/streptomycin antibiotic (all Gibco, Grand Island, NY, USA) and then incubated at $37^{\circ} \mathrm{C}$ in $5 \% \mathrm{CO}_{2}$. When the cells were approximately $80 \%$ confluent, they were trypsinized (Gibco) and halved for characterization by flow cytometry and expansion. EnSCs were seeded into a 24-well plate and transfected with the miR-34a-5p mimics or control miRNAs by Lipofectamine ${ }^{\circledR} 2000$ Transfection Reagent (Invitrogen, Carlsbad, CA, USA).

Flow cytometry. The EnSCs were characterized by flow cytometry for cell surface markers. Cells were incubated with the specific antibody at concentrations recommended by the respective manufacturers for 1 hand analyzed by flow cytometry. The antibodies used were: FITC-conjugated anti-CD146 (eBioScience Inc., San Diego, CA, USA; endometrial stem cell markers), FITC-conjugated anti-CD140b (Abcam, Cambridge, MA, USA; endometrial stem cell markers), FITC-conjugated anti-CD34 (Abcam; hemato-poieticmarker), FITC-conjugated anti-CD31 (Novus Biologicals, Littleton, CO, USA; endothelial marker).

RNA extraction and real-time PCR. To investigate the expression level of VEGFA mRNA and miR-34a-5p, we extracted the total RNA from endometriosis tissues or EnSCs. Tissues or cells were lysed with the Trizol reagent (Life Technologies, Grand Island, NY, USA) according to the manufacturer's specification. mRNAs were dissolved in RNase-free water and stored at $-80^{\circ} \mathrm{C}$ before utilize. The quantitative analysis of RNA was performed by RT-PCR using One Step SYBR PrimeScript PLUS RT-PCT kit (Takara, Tokyo, Japan) for each sample according to the manufacturer's manual. After the reaction, the VEGFA mRNA level and the miR-34a-5p level was calculated and presented as the relative level of VEGFA to $\beta$-actin (as control) by $\Delta \Delta \mathrm{Ct}$ method, each sample was measured for three independent experiments.

Western blot analysis. Western blot analysis was performed to determine the expression of VEGFA on the protein level. Harvested the EnSCs post transfecting, and lysed the cells into cell lysis buffer (Bio-Rad, Hercules, CA, USA). Centrifuged the samples at $12,000 \times \mathrm{g}$ for $15 \mathrm{~min}$ at $4^{\circ} \mathrm{C}$ and collected the supernatant. Protein extracts were boiled in SDS/ $\beta$-mercaptoethanol and separated in a $10 \%$ SDS-PAGE (sodium dodecyl sulphate-polya-crylamide gel electrophoresis). Then, transferred the protein sample to a nitrocellulose membrane (Millipore, Bedford, MA, USA), blocked with $5 \%$ skimmed milk powder overnight at $4^{\circ} \mathrm{C}$. The membrane was incubated with VEGFA-spe-cific antibody in TBST (mouse monoclonal antibody, Abcam, Cambridge, UK, 1:500) at $37^{\circ} \mathrm{C}$ for $1 \mathrm{~h}$, washed with TBST, next incubated with the HRP-linked secondary anti-mouse antibody (New England Biolabs, Ipswich, UK) for $30 \mathrm{~min}$ at $37^{\circ} \mathrm{C}$. ECL kit (Life Science, Woodland Hills, CA, USA) was used to carry out the chemiluminescence reaction. The membrane was scanned by a Smart Chemi-TM lamp Analysis System (Thermo Scientific, Rockford, IL, USA) and quantified according to the band density by Quantity One software with $\beta$-actin as loading control.

Dual luciferase assay. The sequence of 3'UTR of VEGFA from hs-miR-34a-5p were download from Genebank (NC-BI), aligned by Megalign (DNASTAR; GATC Biotech, Konstanz, Germany). The sequence of mutant 3'UTR of VEGFA was synthesized by Sangon Biotech (Shanghai, China). All the sequences in this study were amplified by PCR (polymerase chain reaction) with Phusion polymerase (New England Biolabs). 3'UTR of VEGFA and the mutant 3'UTR of VEGFA were cloned into the upstream of firefly luciferase (Fluc) gene behind the Cytomegalovirus promoter in pmirGLO (Promega, Madison, WI, USA) with restriction endonuclease and DNA ligase (New England Biolabs), received VEGFA-Fluc and mutant-VEGFA-Fluc recombined plasmid. The ESCs seeded in a 24-well plate were co-transfected with the miR-34a-5p mimics/miR-Ctrl and VEGFA-Fluc/mutant-VEGFA-Fluc by Lipofectamine ${ }^{\circledR} 2000$ Transfection Reagent (Invitrogen). $48 \mathrm{~h}$ after transfecting, collected the cells and assayed with the Dual-Luciferase Assay kit (Promega) used GLOMAX (Promega) and recorded data, the relative luciferase activity (\%) represented the expression level of VEGFA.

MTT assay. Transfected EnSCs were diluted to a certain concentration and then were placed over a 96-well plate, at a density of $5 \times 10^{3}$ cells/well. Each well was inoculated with $100 \mu \mathrm{l}$ cell suspension, apart from one blank well (instead with $100 \mu \mathrm{l}$ medium containing $10 \%$ fetal bovine serum). Then they were cultured in a $5 \% \mathrm{CO}_{2}$ incubator at $37^{\circ} \mathrm{C}$. After $0,12,24$ and $48 \mathrm{~h}$ of culture, the proliferation of 

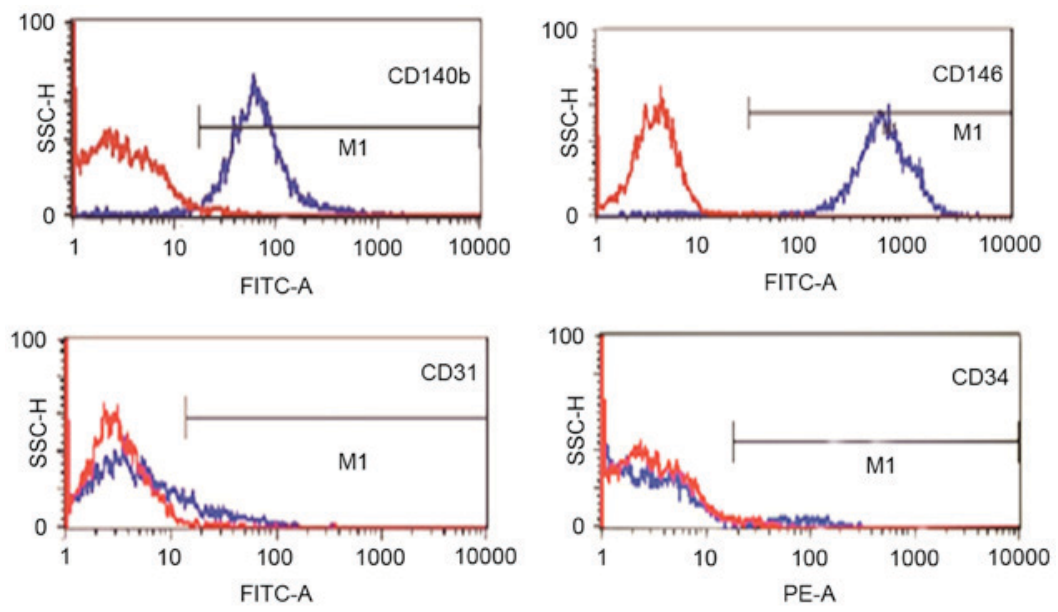

Figure 1. Flow cytometric analysis of isolated EnSCs for EnSCs markers (CD140b, CD146), haemopoietic marker (CD34) and endothelial marker (CD31). As shown the isolated cells are positive for CD140b, CD146 and are negative for CD31 and CD34. EnSCs, endometrial-derived stem cells.

ESCs was detected by MTT assay. With an addition of $20 \mu 1$ 3-(4,5-Dimethylthiazol-2-yl)-5-(3-Carboxymethoxyphenyl)-2(4-Sulfophenyl)-2H-Tetrazolium, Inner Salt (MTS) reagent (Promega) to each well, cells were then cultured in an incubator for 1-4 h. After that, the optical density (OD) at $580 \mathrm{~nm}$ of each well was obtained with a microplate reader (Biotek Instruments, Inc., Vermont, USA). The cell proliferation was evaluated by OD value. All of these experiments were repeated three times.

Statistical analysis. All the analyses were performed using SPSS software, version 17.0 (SPSS Inc., Chicago, IL, USA). SPSS was used to compare miR-34a-5p expression levels between samples taken from patients with or without endometriosis. Data are represented as mean \pm standard deviation (SD) unless indicated otherwise. Statistical significance was tested using Student's t-test, and $\mathrm{P}<0.05$ was considered to indicate a statistically significant difference.

\section{Results}

Characterization of isolated human EnSCs. EnSCs isolated from endometriosis tissue were positive for CD140b, CD146 and negative for CD31 and CD34, as demonstrated by flow cytometry analysis (Fig. 1).

Expression of miR-34a-5p and VEGFA in endometriosis. To investigate the expression of VEGFA in endometriosis, we examined the mRNA level of VEGFA and miR-34a-5p level by quantitative RT-PCR in 10 eutopic endometrial tissues and 10 ectopic endometrial tissues. We focused on miR-34a-5p because it was significantly downregulated miRNA in ectopic endometrial tissues by microarray analysis. It had shown that the relative mRNA level of VEGFA was significantly upregulated in ectopic endometrial tissues than in eutopic endometrial tissues $(\mathrm{P}<0.01)$ (Fig. 2A). The relative level of miR-34a-5p was significantly lower in endometriosis tissues than that in eutopic endometrial tissues $(\mathrm{P}<0.01)$ (Fig. 2B). By analyzing the correlation of relative VEGFA mRNA level with the miR-34a-5p level in ectopic endometrial tissues, we found an inverse correlation between them.
The high mRNA level of VEGFA accompanied a low expression level of miR-34a-5p (Pearson correlation, R2=0.4648, $\mathrm{P}=0.0299$ (Fig. 2C).

Expression of VEGFA in EnSCs after transfection with miR-34a-5p mimics. To further determined whether the expression of VEGFA was downregulated by miR-34a-5p in EnSCs. We examined the relative level of miR-34a-5p and the expression of VEGFA in both mRNA and protein levels after $48 \mathrm{~h}$ transfection. The level of miR-34a-5p was significantly increased after the transfection with miR-34a-5p mimics $(\mathrm{P}<0.01)$, compared with the miR-control group. However, the VEGFA mRNA was reduced post the transfection with miR-34a-5p mimics $(\mathrm{P}<0.01)$. The expression level of VEGFA protein was decreased in EnSCs transfected with miR-34a-5p mimics rather than miR-control group. The band intensity of VEGFA was downregulated in EnSCs transfected with miR-34a-5p mimics $(\mathrm{P}<0.01)$. Therefore, miR-34a-5p suppressed the expression of VEGFA in both mRNA and protein levels in ESCs (Fig. 3).

miR-34a-5p targets the 3'UTR of VEGFA gene. To verify miR-34a-5p inhibited the expression of VEGFA by targeting the 3'UTR of VEGFA, miR-34a-5p mimics and miR-control were transfected into EnSCs which already had been transfected with the 3'UTR of VEGFA linked a luciferase reporter. The results showed that the relative luciferase activity was significantly decreased post transfecting miR-34a-5p mimics $(\mathrm{P}<0.001)$. However, there was no significant difference in the relative luciferase activity between miR-34a-5p mimics and miR-control in the EnSCs, which were transfected with the mutant 3'UTR of VEGFA. All these data demonstrates that miR-34a-5p targets the 3'UTR of VEGFA gene and inhibits the VEGFA expression effectively (Fig. 4).

Effect of miR-34a-5p mimics on the proliferation of EnSCs. miR-34a-5p could modulate the level of VEGFA, which is a regulator of cell growth, so miR-34a-5p may also influence proliferation of EnSCs. Then, we curved the ESCs number at $0,12,24$ and $48 \mathrm{~h}$ after transfecting with miR-control and miR-34a-5p mimics. Fig. 5 showed that the proliferation ability 

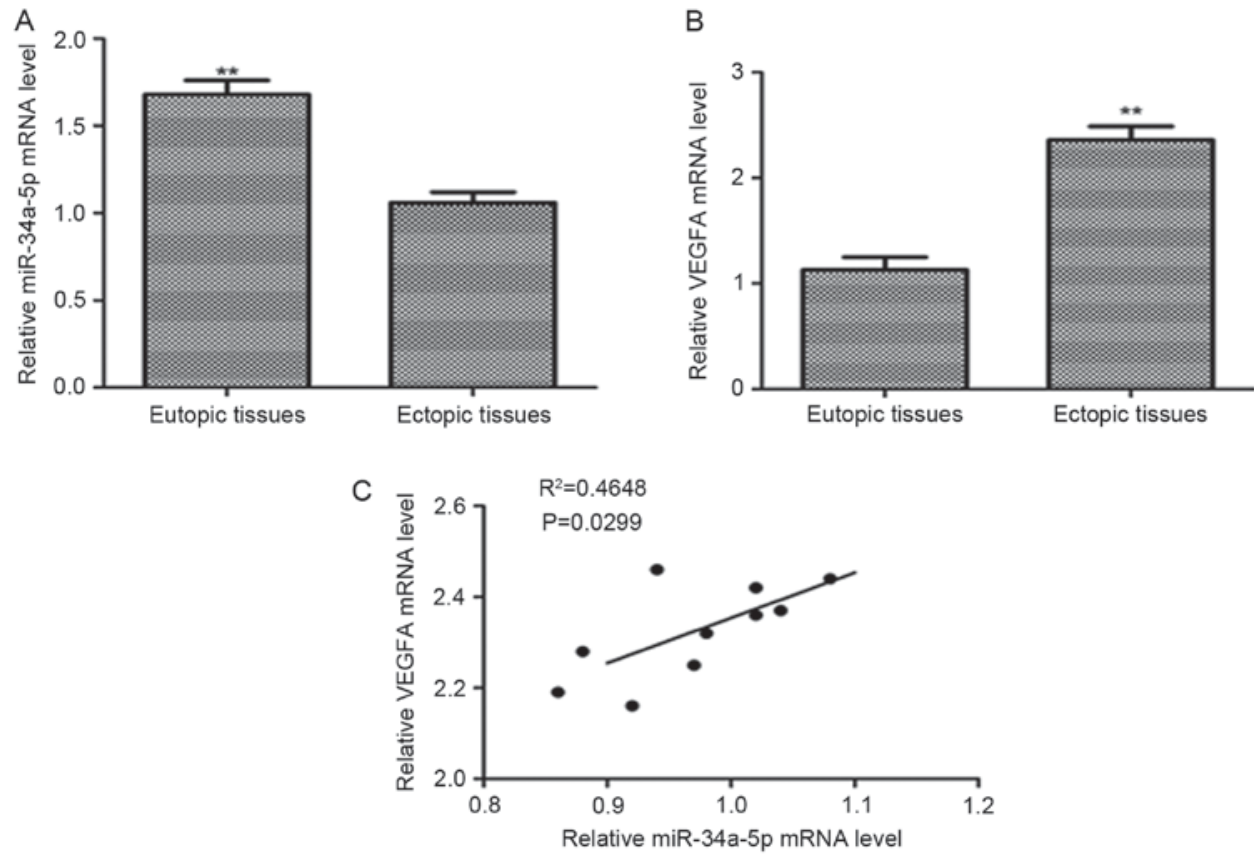

Figure 2. The expression of miR-34a-5p and VEGFA in endometriosis. The relative mRNA level of VEGFA was significantly upregulated in ectopic endometrial tissues than in eutopic endometrial tissues $(\mathrm{P}<0.01)(\mathrm{A})$; The relative level of miR-34a-5p was reduced in endometriosis tissues $(\mathrm{P}<0.01)(\mathrm{B})$; The high mRNA level of VEGFA accompanied a low expression level of miR-34a-5p (Pearson correlation, $\mathrm{R} 2=0.4648, \mathrm{P}=0.0299)(\mathrm{C})$. ${ }^{* *} \mathrm{P}<0.01$ vs. ectopic tissue. miR, microRNA; VEGFA, Vascular endothelial growth factor A.
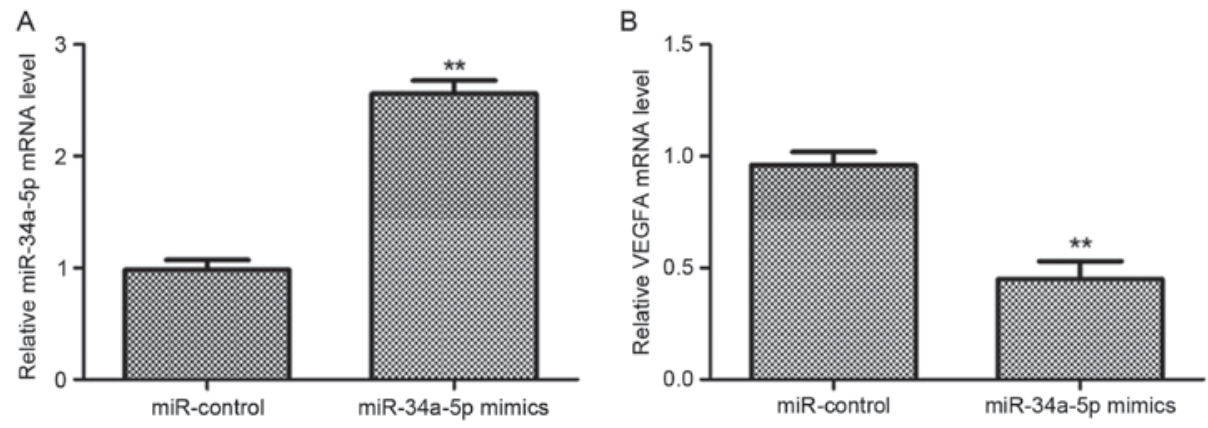

C

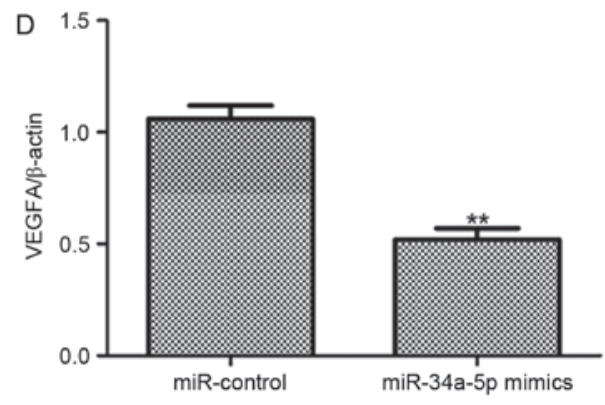

Figure 3. The expression of VEGFA in EnSCs after transfection with miR-34a-5p mimics. The level of miR-34a-5p was significantly increased after the transfection with miR-34a-5p mimics $(\mathrm{P}<0.01)(\mathrm{A})$, compared with the miR-control group; The VEGFA mRNA was reduced post the transfection with miR-34a-5p mimics $(\mathrm{P}<0.01)(\mathrm{B})$; The band intensity of VEGFA was downregulated in EnSCs transfected with miR-34a-5p mimics $(\mathrm{P}<0.01)(\mathrm{C}$ and $\mathrm{D})$. ${ }^{* *} \mathrm{P}<0.01$ vs. miR-control. VEGFA, Vascular endothelial growth factor A; EnSCs, endometrial-derived stem cells; miR, microRNA.

of EnSCs was significantly reduced post the transfection with miR-34a-5p mimics $(\mathrm{P}<0.01)$.

\section{Discussion}

The present study is the first to investigate the expression of miR-34a-5p and VEGFA in endometriosis. The results showed that miR-34a-5p expression levels significantly decreased in endometriosis compared with that in without endometriosis patients, but the VEGFA expression levels significantly increased in endometriosis compared with without endometriosis patients. Negative correlation was observed between relative VEGFA mRNA level and miR-34a-5p level in ectopic endometrial tissues. Moreover, over-expression of miR-34a-5p 
3'UTR wild-type

$$
\begin{array}{r}
\text { VEGFA...... 5'-GUUAUUGCUCACUGGA-3' } \\
\text { miR-34a-5p .......3'-CUGUUCAGUGUGACCG-5' }
\end{array}
$$

3'UTR mutant

VEGFA ...... 5' -GUUAUUGCUGUGACCA-3'

miR-34a-5p ...... 3' -CUGUUCAGUGUGACCG-5'
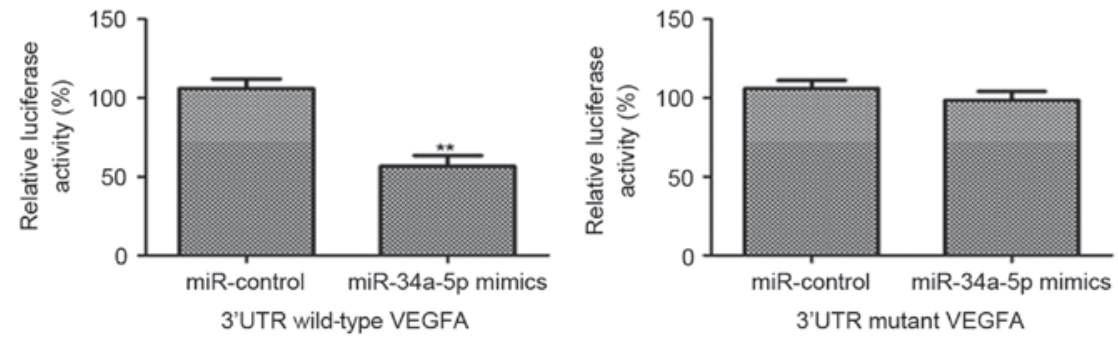

Figure 4. miR-34a-5p targets the 3'UTR of VEGFA gene. The relative luciferase activity was significantly decreased post transfecting miR-34a-5p mimics $\left({ }^{* *} \mathrm{P}<0.01\right.$ vs. miR-control); However, there was no significant difference in the relative luciferase activity between miR-34a-5p mimics and miR-control in the EnSCs, which were transfected with the mutant 3'UTR of VEGFA (P>0.05). miR, microRNA; VEGFA, Vascular endothelial growth factor A; UTR, untranslated region; EnSCs, endometrial-derived stem cells.

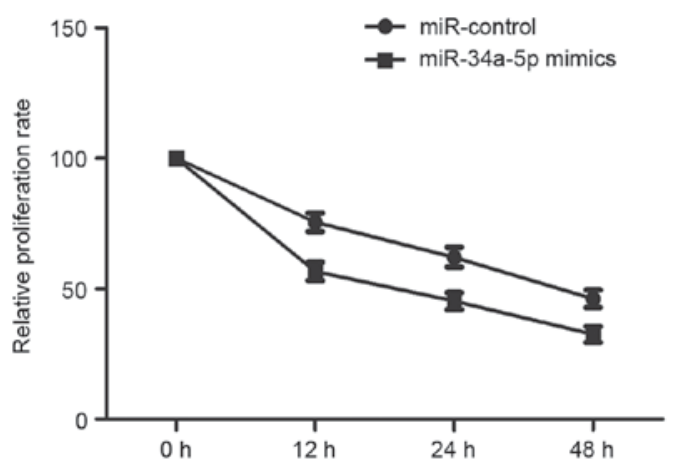

Figure 5. Effect of miR-34a-5p mimics on the proliferation of ESCs. The proliferation ability of EnSCs was significantly reduced post the transfection with miR-34a-5p mimics, compared with miR-control group $(\mathrm{P}<0.01)$. miR, microRNA; ESC, embryonic stem cells; EnSCs, endometrial-derived stem cells.

can significantly inhibit the expression and proliferation of VEGFA in EnSCs.

miR-34a belongs to a miRNA family, and its as a strong antitumor regulator of cell growth in multiple myeloma was observed $(16,17)$. miR-34a is, to date, one of the most characterized tumor suppressor miRNAs in a variety of tumors (18). miR-34a-5p expression has been shown to be directly regulates the expression of proteins involved in cell cycle, differentiation, apoptosis, and antagonizes processes, including cervical, ovarian and testicular cancer $(19,20)$. Ma et al conducted a series of studies on miR-34a-5p, and were the first to confirm its pro-apoptotic function (21). Moreover, miR-34a-5p has been found to inhibit cell proliferation and invasion in vitro, which suggested that miR-34a-5p might play a role in inhibiting tumor recurrence (22). miR-34a-5p has been reported to be a direct transcriptional target of p53 and is downregulated in various types of tumors (23). We measured miR-34a-5p expression levels in endometrial tissue and ESCs. Regardless of the sample types, miR-34a-5p expression levels were consistently downregulated in patients with endometriosis. The results support previous reports that miR-34a-5p suppresses disease progression, including diseases associated with the endometrium.

VEGFA is a dimeric glycoprotein that plays an important role in vasculogenesis, and its overexpression often occurred in various cancers (24). VEGF is involved in the pathogenesis of endometriosis. Blocking VEGF to treat endometriosis decreases vascular density and cell proliferation and increases cell apoptosis (25). In this study, we compared the expression level of VEGFA in eutopic and ectopic endometrial tissues. The results showed that the VEGFA was overexpressed in ectopic endometrial tissues compared with eutopic endometrial tissues. It had reported that miRNAs could regulate the expression of VEGFA (26). miRNA-34a modulates the phosphorylation of FAK by negatively regulating VEGF in colorectal cancer (27). miR-638 expression was inversely correlated with VEGF expression in human hepatocellular carcinoma samples (28). In addition, miR-3072-5p inhibited VEGF expression in ischemic preconditioning was also observed (29). Here we demonstrated that miR-34a-5p regulates cell proliferation and angiogenesis by regulating VEGFA levels in EnSCs, and VEGFA mRNA level was significantly increased while the level of miR-34a-5p was markedly decreased in endometriosis tissues. The correlation analysis showed that the VEGFA mRNA level was inversely correlated with miR-34a-5p. All these data indicated a potential role of miR-34a-5p in inhibiting expression of VEGFA in endometriosis.

Angiogenesis is a crucial determinant in tumor initiation, progression, and metastasis. Angiogenesis plays an important role in endometriosis progression (30). VEGFA is thought to be the primary stimulator of angiogenesis, during the course of development and in a variety of pathological conditions. A large number of researches have been done showing that miRNAs play important roles in vascular development and angiogenesis (31). It is reported that miRNAs (eg miR-17-5p 
and miR-199a-5p) regulate angiogenesis (32). In addition, miR-203 suppresses tumor growth and angiogenesis by targeting VEGFA in cervical cancer (33). We found an inverse correlation between levels of VEGFA and miR-34a-5p. To our knowledge, this is the first demonstration that VEGFA is a functional target of miR-34a-5p. As such, miR-34a-5p regulates cell proliferation and angiogenesis through its effect on VEGFA.

In summary, VEGFA was overexpressed in endometriosis tissues, and we identified miR-34a-5p is able to target the VEGF gene, preventing the latter to function as an inhibitor of angiogenesis. These results imply that miR-34a-5p might regulate VEGFA in ESCs and might contribute to the pathogenesis of endometriosis. This study may provide a potential biomarker for endometriosis therapeutics.

\section{Acknowledgements}

The present study was supported by the Science and Technology Planning Project of Guangdong Province (grant no. 2014A020212667) and the National Natural Science Fundation of China (grant no. 81701418).

\section{References}

1. Yoder N, Tal R and Martin JR: Abdominal ectopic pregnancy after in vitro fertilization and single embryo transfer: A case report and systematic review. Reprod Biol Endocrinol 14: 69, 2016.

2. Bulun SE: Endometriosis. N Engl J Med 360: 268-279, 2009.

3. Dinulescu DM, Ince TA, Quade BJ, Shafer SA, Crowley D and Jacks T: Role of K-ras and Pten in the development of mouse models of endometriosis and endometrioid ovarian cancer. Nat Med 11: 63-70, 2005.

4. Greene AD, Lang SA, Kendziorski JA, Sroga-Rios JM, Herzog TJ and Burns KA: Endometriosis: Where are we and where are we going? Reproduction 152: R63-R78, 2016

5. Maruyama T and Yoshimura Y: Stem cell theory for the pathogenesis of endometriosis. Front Biosci 4: 2754-2763, 2012.

6. Sasson IE and Taylor HS: Stem cells and the pathogenesis of endometriosis. Ann N Y Acad Sci 1127: 106-115, 2008.

7. Kao AP, Wang KH, Chang CC, Lee JN, Long CY, Chen HS, Tsai CF, Hsieh TH and Tsai EM: Comparative study of human eutopic and ectopic endometrial mesenchymal stem cells and the development of an in vivo endometriotic invasion model. Fertil Steril 95: 1308-1315, 2011.

8. Groothuis PG, Nap AW, Winterhager E and Grümmer R: Vascular development in endometriosis. Angiogenesis 8: 147-156, 2005.

9. Teague EM, Print CG and Hull ML: The role of microRNAs in endometriosis and associated reproductive conditions. Hum Reprod Update 16: 142-165, 2010.

10. Zou Y, Guo CG and Zhang MM: Inhibition of human hepatocellular carcinoma tumor angiogenesis by siRNA silencing of VEGF via hepatic artery perfusion. Eur Rev Med Pharmacol Sci 19: 4751-4761, 2015.

11. Liu B, Ding JF, Luo J, Lu L, Yang F and Tan XD: Seven protective miRNA signatures for prognosis of cervical cancer. Oncotarget 7: 56690-56698,2016.

12. Cho S, Mutlu L, Grechukhina O and Taylor HS: Circulating microRNAs as potential biomarkers for endometriosis. Fertil Steril 103: 1252-1260, 2015.

13. Toloubeydokhti T, Pan Q, Luo X, Bukulmez O and Chegini N: The expression and ovarian steroid regulation of endometrial micro-RNAs. Reprod Sci 15: 993-1001, 2008.

14. Braza-Boïls A, Salloum-Asfar S, Marí-Alexandre J, Arroyo AB, González-Conejero R, Barceló-Molina M, García-Oms J, Vicente V, Estellés A, Gilabert-Estellés and Martínez C: Peritoneal fluid modifies the microRNA expression profile in endometrial and endometriotic cells from women with endometriosis. Hum Reprod 30: 2292-2302, 2015.
15. Gao J, Li N, Dong Y, Li S, Xu L, Li X, Li Y, Li Z, Ng SS, Sung JJ, et al: miR-34a-5p suppresses colorectal cancer metastasis and predicts recurrence in patients with stage II/III colorectal cancer. Oncogene 34: 4142-4152, 2015.

16. Scognamiglio I, Di Martino MT, Campani V, Virgilio A, Galeone A, Gullà A, Gallo Cantafio ME, Misso G, Tagliaferri P, Tassone P, et al: Transferrin-conjugated SNALPs encapsulating 2'-O-methylated miR-34a for the treatment of multiple myeloma. Biomed Res Int. Biomed Res Int 2014: 217365, 2014.

17. Di Martino MT, Campani V, Misso G, Gallo Cantafio ME, Gullà A, Foresta U, Guzzi PH, Castellano M, Grimaldi A, Gigantino V, et al: In vivo activity of miR-34a mimics delivered by stable nucleic acid lipid particles (SNALPs) against multiple myeloma. PLoS One 9: e90005, 2014.

18. Misso G, Di Martino MT, De Rosa G, Farooqi AA, Lombardi A, Campani V, Zarone MR, Gullà $\mathrm{A}$, Tagliaferri $\mathrm{P}$, Tassone $\mathrm{P}$ and Caraglia M: Mir-34: A new weapon against cancer? Mol Ther Nucleic Acids 3: e194, 2014.

19. Song P, Ye LF, Zhang C, Peng T and Zhou XH: Long non-coding RNA XIST exerts oncogenic functions in human nasopharyngeal carcinoma by targeting miR-34a-5p. Gene 592: 8-14, 2016.

20. Li XY, Wen JY, Jia CC, Wang TT, Li X, Dong M, Lin QU, Chen ZH, Ma XK, Wei LI, et al: MicroRNA-34a-5p enhances sensitivity to chemotherapy by targeting AXL in hepatocellular carcinoma MHCC-97 L cells. Oncol Lett 10: 2691-2698, 2015.

21. Ma ZB, Kong XL, Cui G, Ren CC, Zhang YJ, Fan SJ and Li YH: Expression and clinical significance of miRNA-34a in colorectal cancer. Asian Pac J Cancer Prev 15: 9265-9270, 2014.

22. Walton SJ, Lewis A, Jeffery R, Thompson H, Feakins R, Giannoulatou E, Yau C, Lindsay JO, Clark SK and Silver A: Familial adenomatous patients with desmoid tumours show increased expression of miR-34a in serum and high levels in tumours. Oncoscience 3: 173-185, 2016

23. Lu H, Hao L, Li S, Lin S, Lv L, Chen Y, Cui H, Zi T, Chu X, Na L and Sun C: Elevated circulating stearic acid leads to a major lipotoxic effect on mouse pancreatic beta cells in hyperlipidaemia via a miR-34a-5p-mediated PERK/p53-dependent pathway. Diabetologia 59: 1247-1257, 2016.

24. Goel HL and Mercurio AM: VEGF targets the tumour cell. Nat Rev Cancer 13: 871-882, 2013.

25. Li YZ, Wang LJ, Li X, Li SL, Wang JL, Wu ZH, Gong L and Zhang XD: Vascular endothelial growth factor gene polymorphisms contribute to the risk of endometriosis: An updated systematic review and meta-analysis of 14 case-control studies. Genet Mol Res 12: 1035-1044, 2013.

26. Ramón LA, Braza-Boïls A, Gilabert-Estellés J, Gilabert J, España F, Chirivella $M$ and Estellés A: MicroRNAs expression in endometriosis and their relation to angiogenic factors. Hum Reprod 26: 1082-1090, 2011.

27. Zhang D, Zhou J and Dong M: Dysregulation of microRNA-34a expression in colorectal cancer inhibits the phosphorylation of FAK via VEGF. Dig Dis Sci 59: 958-967, 2014

28. Cheng J, Chen Y, Zhao P, Liu X, Dong J, Li J, Huang C, Wu R and Lv Y: Downregulation of miRNA-638 promotes angiogenesis and growth of hepatocellular carcinoma by targeting VEGF. Oncotarget 7: 30702-30711, 2016.

29. Ueno K, Samura M, Nakamura T, Tanaka Y, Takeuchi Y, Kawamura D, Takahashi M, Hosoyama T, Morikage N and Hamano K: Increased plasma VEGF levels following ischemic preconditioning are associated with downregulation of miRNA-762 and miR-3072-5p. Sci Rep 6: 36758, 2016.

30. Kim SH, Choi YM, Chae HD, Kim CH and Kang BM: Decreased expression of angiogenin in the eutopic endometrium from women with advanced stage endometriosis. J Korean Med Sci 23: 802-807, 2008.

31. Finn NA and Searles CD: Intracellular and extracellular miRNAs in regulation of angiogenesis signaling. Curr Angiogenes 4: 299-307, 2012.

32. Hsu CY, Hsieh TH, Tsai CF, Tsai HP, Chen HS, Chang Y, Chuang HY, Lee JN, Hsu YL and Tsai EM: miRNA-199a-5p regulates VEGFA in endometrial mesenchymal stem cells and contributes to the pathogenesis of endometriosis. J Pathol 232: 330-343, 2014.

33. Zhu X, Er K, Mao C, Yan Q, Xu H, Zhang Y,Zhu J, Cui F, Zhao W and Shi H: miR-203 suppresses tumor growth and angiogenesis by targeting VEGFA in cervical cancer. Cell Physiol Biochem 32: 64-73, 2013. 